\title{
Avaliação nutricional de pacientes com câncer de mama atendidas no Serviço de Mastologia do Hospital das Clínicas, Belo Horizonte (MG), Brasil
}

\author{
Nutritional status of patients with breast cancer attended \\ in the Mastology Service of Belo Horizonte's Hospital das Clínicas \\ in the state of Minas Gerais, Brazil
}

\author{
Dirce Ribeiro de Oliveira ${ }^{1}$ \\ Erika Simone Coelho Carvalho ${ }^{2}$ \\ Liliane Cunha Campos ${ }^{3}$ \\ José Adalberto Leal ${ }^{2}$ \\ Estela Viana Sampaio ${ }^{3}$ \\ Geovanni Dantas Cassali ${ }^{3}$
}

${ }^{1}$ Escola de Enfermagem, Universidade Federal de Minas Gerais. Av. Professor Alfredo Balena, Santa Efigênia. 30.130-100 Belo Horizonte MG Brasil. dirceribeiroliveira@gmail.com

${ }^{2}$ Instituto de Previdência dos Servidores do Estado de Minas Gerais.

${ }^{3}$ Universidade Federal de Minas Gerais.

\begin{abstract}
The scope of this article is to evaluate the nutritional status, body composition and tumor characteristics of 31 patients with breast cancer attended at the Mastology Service of Hospital das Clinicas of the Federal University of Minas Gerais. Dietary data were obtained from the 24hour dietary recall in the pre-operative state and analyzed by the DietWin Professional $2008 \mathrm{Nu}$ trition Software. Body composition was assessed by bioelectrical impedance analysis. The tumor characterization data were collected from medical records. The mean age of patients was $50.75 \pm$ 14.34 years. Excess body weight was found in $58 \%$ and waist circumference greater than $80 \mathrm{~cm}$ in 64.5\% of patients Excessive consumption of oils and sugars was observed in $90.3 \%$ and $83.8 \%$, respectively. Most had low intakes of calcium and vitamins B6, B12 and A. The predominant diagnosis was type II histological grade invasive ductal carcinoma in stage II or III. The prevalence of overweight and inadequate dietary intake demonstrate the need for individualized nutritional guidance and monitoring to improve the prognosis and quality of the life of patients.
\end{abstract}

Key words Breast neoplasms, Nutrition surveys, Obesity, Body composition
Resumo O objetivo deste artigo é avaliar o perfil nutricional, a composição corporal e as características tumorais de 31 pacientes com câncer de mama atendidas pelo Serviço de Mastologia do Hospital das Clínicas da Universidade Federal de Minas Gerais. Os dados da dieta foram coletados usando-se o recordatório de 24 horas no momento pré-cirúrgico e analisados pelo Software de Nutrição DietWin Profissional 2008. A composição corporal foi avaliada por análise de impedância bioelétrica. A caracterização tumoral foi realizada através da avaliação dos dados obtidos no prontuário de cada paciente. A idade média foi de 50,75 \pm 14,3 anos. Foi observado excesso de peso corporal em $58 \%$ das pacientes e circunferência da cintura maior que $80 \mathrm{~cm}$ em $64,5 \%$. Consumo excessivo de alimentos pertencentes aos grupos dos óleos e dos açúcares foi verificado em 90,3\% e 83,8\%, respectivamente. A maioria das pacientes apresentou baixo consumo de cálcio e de vitaminas B6, B12 e A. O diagnóstico predominante foi de carcinoma ductal invasivo, de grau histológico tipo II em estadio II ou III. A prevalência do excesso de peso e a inadequação do consumo alimentar demonstram a necessidade de orientação e acompanhamento nutricional individualizado, visando melhorar o prognóstico e a qualidade de vida da paciente.

Palavras-chave Neoplasias da mama, Inquéritos nutricionais, Obesidade, Composição corporal 


\section{Introdução}

A obesidade tem um efeito prognóstico adverso na sobrevida das mulheres com câncer de mama. O excesso de peso corporal parece influenciar no desenvolvimento e na progressão do câncer de mama devido ao aumento da síntese do estrógeno, resistência à insulina e ativação de vias inflamatórias ${ }^{1}$.

Estudos sugerem associações entre o peso corporal, índice de massa corporal (IMC) e circunferência da cintura como fatores de risco para o desenvolvimento de câncer de mama ${ }^{2}$. Além disso, o estresse causado pelo diagnóstico e tratamento quimioterápico do câncer de mama tem sido relacionado com ganho de peso devido à mudança no estilo de vida, caracterizado por inatividade física e hábitos alimentares inadequados ${ }^{3,4}$

Fatores dietéticos são identificados na gênese e progressão da doença. Alguns componentes alimentares possuem efeito preventivo, agindo através de variados mecanismos de ação e atuando no bloqueio da carcinogênese. Entre eles destacam-se as fibras, as vitaminas e os minerais, os fitoquímicos e os ácidos graxos poliinsaturados, o ácido graxo ômega 3 e o ácido linoleico conjugado $^{5}$.

A adesão à dieta do tipo mediterrânea foi associada com redução moderada do risco de câncer de mama em mulheres na pós-menopausa, principalmente no risco do desenvolvimento de tumores negativos para receptores hormonais ${ }^{6}$. Dados de estudos observacionais (coorte e casocontrole) sugerem que as principais associações entre dieta e câncer de mama envolvem o consumo de vitamina D, cálcio, gordura e álcool, e são encontradas em mulheres adultas na pré-menopausa ${ }^{7}$.

Entre mulheres que consumiam álcool, mesmo esporadicamente, o risco de contrair câncer de mama foi 1,5 vezes maior em relação àquelas que se abstinham, sugerindo três mecanismos de ação do álcool no desenvolvimento do câncer de mama: aumento dos níveis de estrógenos e de seus receptores, liberação de produtos do metabolismo do álcool e menor ingestão de nutrientes essenciais pelos pacientes alcoólatras ${ }^{8}$.

Conhecer o perfil nutricional das pacientes acometidas pelo câncer torna-se uma medida importante no desenvolvimento de ações multiprofissionais a fim de elaborar estratégias direcionadas às diferentes fases da doença e do tratamento ${ }^{9}$. É preciso individualizar a terapia, prevenir as complicações nutricionais decorrentes do tratamento e da doença, dando ao paciente, um melhor suporte nutricional e garantindo melhor qualidade de vida.

Dessa forma, o presente trabalho objetivou avaliar o perfil nutricional, a composição corporal, as características tumorais e as possíveis associações entre variáveis de pacientes com câncer de mama atendidas pelo Serviço de Mastologia do Hospital das Clínicas da Universidade Federal de Minas Gerais.

\section{Pacientes e métodos}

Trata-se de um estudo transversal desenvolvido no Serviço de Mastologia do Hospital das Clínicas da UFMG (Belo Horizonte, MG), no período de maio a agosto de 2011. O estudo foi aprovado pelo Comitê de Ética em Pesquisa da UFMG. Cada paciente que concordou em participar da pesquisa assinou o termo de consentimento livre e esclarecido.

A amostra de conveniência foi composta por 31 mulheres com diagnóstico recente de câncer de mama. Os critérios de exclusão foram: gravidez, uso de marcapasso, e tratamento por rádio ou quimioterápico neoadjuvante.

$\mathrm{Na}$ análise antropométrica e de composição corporal excluíram-se pacientes com edema e transtornos psiquiátricos, por não serem capazes de responder aos inquéritos alimentares. Dos 31 prontuários consultados, um não continha informações sobre tipo e estadiamento do tumor e dois não informaram o grau histológico. Foram utilizados dados de imuno-histoquímica para receptores hormonais (estrógeno e progesterona) relatados em 20 prontuários e para HER2 em 18 prontuários.

Aplicou-se um questionário desenvolvido para a pesquisa, onde foram avaliados os seguintes fatores de risco não modificáveis: nuliparidade; idade ao nascimento do primeiro filho (acima de 30 anos); idade da menarca (menor que 12 anos); idade da menopausa (acima de 55 anos); uso de anticoncepcionais; uso da terapia de reposição hormonal para menopausa e tempo de amamentação inferior a seis meses. Os fatores modificáveis avaliados foram: prática de atividade física (informada pela paciente); composição corporal e consumo alimentar, ambos aferidos $^{10}$

Os dados da dieta foram coletados usandose o recordatório de 24 horas (R24h), com auxílio do álbum de registro fotográfico ${ }^{11}$ para estimar o consumo alimentar, e o Questionário de Frequência do Consumo Alimentar (QFCA) ${ }^{12}$. 
A qualidade da dieta foi avaliada pelo Índice de Alimentação Saudável Adaptado (IASad) para a população brasileira ${ }^{13}$. Este consiste em analisar os alimentos relatados com base no questionário de frequência alimentar, onde os alimentos são convertidos em porções pelo valor energético e classificados nos grupos aos quais pertencem: cereais, frutas, hortaliças, leguminosas, produtos lácteos, carnes, doces e açúcares, óleos e gorduras. Outros nutrientes analisados pelo índice são gordura total, saturada e colesterol. A partir da pontuação obtida das variáveis analisadas pelo IASad, as dietas foram classificadas como: de boa qualidade (superior a 100 pontos), precisando de melhorias (71-100 pontos) e de má qualidade (inferior a 71 pontos). As análises do consumo alimentar foram realizadas no Software de Nutrição DietWin Profissional 2008, que foi alimentado com diferentes fontes para informação da composição dos alimentos ${ }^{14-16}$. Foram considerados os nutrientes com recomendação nutricional determinada pela DRI (Dietary Reference Intakes $)^{17}$.

Foram aferidos peso e altura, calculado o Índice de Massa Corporal (IMC) e adotados os pontos de corte recomendados pela Organização Mundial de Saúde (OMS) ${ }^{18}$. As circunferências da cintura (CC) e do quadril (CQ) foram aferidas e calculou-se a relação cintura-quadril (RCQ). Foram considerados os pontos de corte para classificação de gordura visceral aumentada, valores de CC e" 80 cm (nível I) e e" $88 \mathrm{~cm}$ (nível II) e para determinação do risco de doença cardiovascular utilizou-se o valor de RCQ e" $0,85^{19}$. A espessura das pregas cutâneas tricipital (PCT), bicipital (PCB), subescapular (PCSE) e suprailíaca (PCSI) foram aferidas no hemicorpo direito das pacientes. Estimou-se o percentual de gordura corporal (\% GC) através dos valores adotados por Durmin e Womersley ${ }^{20}$ e classificados de acordo com os valores propostos por Lohman ${ }^{21}$. A composição corporal foi avaliada por Análise de Impedância Bioelétrica (BIA), utilizando-se o pletismógrafo Biodynamics ${ }^{\circledR}$ "BIA 310e".

As análises estatísticas foram realizadas com auxílio do software Grap Pad Instat v.5. Foram realizadas análises de frequência para avaliar os dados nutricionais e antropométricos e as características sociodemográficas. Os dados foram avaliados quanto à normalidade pelo teste D'Agostino \& Pearson e submetidos à análise de variância ANOVA. Os dados não paramétricos foram avaliados pelo teste Mann Whitney. Possíveis correlações foram avaliadas pelos testes de Pearson e Spearman. Foram consideradas como significativas as associações e as concordâncias cuja probabilidade de significância do teste foi $\mathrm{p}$ $<0,05$.

\section{Resultados}

A idade das pacientes variou entre 29 e 92 anos (média 50,75 \pm 14,34). Observou-se ausência de analfabetismo e $56,26 \%$ relataram ter ensino fundamental completo. Das participantes, $81,25 \%$ eram economicamente ativas.

Dos fatores de risco avaliados, verificou-se que $83,9 \%$ (26/31) das pacientes não fumavam; todas eram sedentárias, 69\% (22/31) tinham de um a cinco filhos, $77 \%$ tiveram filhos com menos de 30 anos e $75 \%$ menarca com idade igual ou superior a 12 anos. Quanto à terapia de reposição hormonal, $80 \%(12 / 15)$ relataram a não utilização e 64,5\% (20/31) eram adeptas do uso de contraceptivo oral.

Quanto à caracterização tumoral, o diagnóstico predominante foi de carcinoma ductal invasivo $(22 / 31 ; 70,9 \%)$, de grau histológico tipo II $(44,8 \%)$ em estádio II ou III (ambos 28,1\%). O perfil imuno-histoquímico foi variado, como pode ser observado na Tabela 1.

Tabela 1. Frequência do tipo, graduação, estadiamento e perfil imunohistoquímico dos tumores das pacientes atendidas pelo Serviço de Mastologia do Hospital das Clínicas, da UFMG, nos anos 2010-2011.

\begin{tabular}{|c|c|}
\hline Parâmetros & n $(\%)$ \\
\hline \multicolumn{2}{|l|}{ Tipo histológico ${ }^{\mathrm{a}}$} \\
\hline Carcinoma ductal invasor & $22(70,9 \%)$ \\
\hline Outros & $9(29,0 \%)$ \\
\hline \multicolumn{2}{|l|}{ Graduação histológica $^{\mathrm{b}}$} \\
\hline Pouco diferenciado & $5(17,2 \%)$ \\
\hline Moderadamente diferenciado & $13(44,8 \%)$ \\
\hline Muito diferenciado & $11(37,9 \%)$ \\
\hline \multicolumn{2}{|l|}{ Estadiamento $^{c}$} \\
\hline 0 & $2(6,6 \%)$ \\
\hline I & $4(13,3 \%)$ \\
\hline IIA/ IIB & $9(30,0 \%)$ \\
\hline IIIA/ IIIB/ IIIC & $9(30,0 \%)$ \\
\hline IV & $6(20,0 \%)$ \\
\hline \multicolumn{2}{|l|}{ Perfil imunohistoquímico $^{\mathrm{d}}$} \\
\hline $\mathrm{RE}+$ & $12(60 \%)$ \\
\hline $\mathrm{RP}+$ & $10(50 \%)$ \\
\hline Her-2 - & $10(55,5 \%)$ \\
\hline
\end{tabular}

${ }^{\text {a }}$ Total de 31 pacientes informados; btotal de 29 pacientes informados; ${ }^{\mathrm{c}}$ total de 30 pacientes informados; ${ }^{\mathrm{d}} \mathrm{RE} / \mathrm{RP}$ total de 20 pacientes informados, HER-2 total de 18 pacientes. 
A avaliação do estado nutricional mostrou que $58 \%$ das mulheres apresentaram excesso de peso corporal, variando de 35 a $116 \mathrm{Kg}$ (média $66 \mathrm{Kg} \pm 16)$; 93,5\% (29/31) mostraram excesso de gordura corporal; 64,5\% (20/31) apresentaram valores de CC maior que $80 \mathrm{~cm}$, enquanto $35,5 \%(11 / 31)$ os tinham adequados $(\mathrm{p}<0,001)$ (Tabela 2). A RCQ estava aumentada em 48,39\% das pacientes. Excesso de gordura corporal (> $32 \%$ ) foi encontrado em $93,55 \%$ das pacientes quando avaliadas pelas medidas das pregas cutâneas, enquanto que pela BIA 54,8\% das avaliadas tiveram este diagnóstico. Foi observada correlação positiva entre IMC e \%GC (r2 = 0,79; p < $0,0001)$, IMC e CC ( $\mathrm{r} 2=0,84 ; \mathrm{p}<0,001)$, CC e $\% \mathrm{GC}(\mathrm{r} 2=0,58 ; \mathrm{p}<0,001)$.

Das possíveis associações avaliadas entre o estado menopausal e CC, IMC, \%GC, IAS, estadiamento, tipo e grau histológico do tumor, verificou-se que pacientes na pós-menopausa apresentaram estadiamento mais avançado ( $\mathrm{p}=$ 0,001).

O índice de alimentação saudável e seus componentes evidenciaram um padrão dietético de má qualidade como pode ser observado na Tabela 3. Os resultados do IASad, mostraram que 87\% das pacientes tinham alimentação de "má qualidade" (pontuação < 71) e nenhuma apresentou alimentação considerada de "boa qualidade" (pontuação $\geq 100$ ). A maioria consumiu quantidades de porções abaixo do recomendado

Tabela 2. Dados antropométricos e composição corporal de mulheres com câncer de mama atendidas pelo Serviço de Mastologia do Hospital das Clínicas, da UFMG, nos anos 2010-2011.

\begin{tabular}{lr}
\hline \multicolumn{1}{c}{ Parâmetros } & \multicolumn{1}{c}{$\mathbf{n}(\%)^{\mathbf{a}}$} \\
\hline Peso $(\mathrm{Kg})$ & \\
$\quad<60$ & $12(38,7 \%)$ \\
$\quad 60-70$ & $7(22,5 \%)$ \\
$\quad>70$ & $12(38,7 \%)$ \\
$\%$ gordura corporal & \\
$\quad<30$ & $2(6,4 \%)$ \\
$30-35$ & $4(12,5 \%)$ \\
$\geq 35$ & $25(80,6 \%)$ \\
Circunferência da cintura $(\mathrm{cm})$ & \\
$\quad<80$ & $11(35,5 \%)$ \\
$\geq 80$ & $20(64,5 \%)$ \\
IMC $\left(\right.$ Kg/m $\left.{ }^{2}\right)$ & \\
$<25$ & $13(41,91 \%)$ \\
$25-29$ & $9(29 \%)$ \\
$\geq 30$ & $9(29 \%)$ \\
\hline
\end{tabular}

${ }^{\text {a dados de }} 31$ pacientes.
Tabela 3. Frequência do IAS e seus componentes, adaptado de acordo com as recomendações do Guia Alimentar da População Brasileira, após análise do recordatório alimentar (R24h) de mulheres com câncer de mama atendidas pelo Serviço de Mastologia do Hospital das Clínicas, da UFMG, nos anos 2010-2011.

\begin{tabular}{lr}
\hline \multicolumn{1}{c}{ Componentes } & n (\%) \\
\hline IASad $^{\mathrm{a}}($ Escore $)$ & \\
$<71$ & $27(87 \%)$ \\
$71-100$ & $4(83,8 \%)$ \\
$>100$ & $0(0 \%)$
\end{tabular}

Grupo do arroz, pães, massas, batata, mandioca (Porções)

$$
<5 \quad 24(77,4 \%)
$$$$
\geq 5
$$

$7(22 \%)$

Grupo das verduras e legumes

(Porções)

$$
\begin{array}{cr}
<4 & 29(93,5 \%) \\
\geq 4 & 2(6,4 \%) \\
\text { Grupo das frutas (Porções) } & \\
\quad<3 & 26(83,8 \%) \\
\quad \geq 3 & 5(16,1 \%) \\
\text { Grupo de feijões (Porções) } & \\
\quad<1 & 9(29,0 \%) \\
\geq 1 & 22(70,9 \%)
\end{array}
$$

Grupo das carnes (Porções)

$$
\begin{array}{lr}
<1 & 5(16,1 \%) \\
\geq 1 & 26(83,8 \%)
\end{array}
$$

Grupo dos laticínios (Porções)

$<3$

$25(80,6 \%)$

$6(19,3 \%)$

Grupo dos óleos e gorduras (Porções)

$<1$

$3(9,7 \%)$

$\geq 1$

Grupo dos açúcares e doces (Porções)

$<1$

$28(90,3 \%)$

$>1$

$5(16,1 \%)$

$26(83,9 \%)$

Gordura total (\%)

$<30 \%$

$18(58,1 \%)$

$\geq 30 \%$

$13(41,9 \%)$

Gordura saturada (\%)

$<10 \% \mathrm{VCT}$

$19(61,2 \%)$

$\geq 10 \% \mathrm{VCT}$

$12(38,8 \%)$

Colesterol (mg)

$<300$

$22(70,9 \%)$

$\geq 300$

$9(29,0 \%)$

Variedade (n)

$<8$

$6(19,3 \%)$

$\geq 8$

$25(80,6 \%)$

Água $(\mathrm{L})^{\mathrm{b}}$

$<2$

$30(96,8 \%)$

$\geq 2 \quad 1(6,2 \%)$

Dados de acordo com as recomendações do Guia Alimentar da População Brasileira, após análise do recordatório alimentar (R24h) de 31 mulheres com câncer de mama. ${ }^{\mathrm{a}}$ IASad= Índice de alimentação saudável adaptado. ${ }^{b}$ total de 31 pacientes. 
de alimentos pertencentes ao grupo do arroz, pães, massas, batata, mandioca, grupo das verduras e legumes, grupo das frutas e grupo dos laticínios. Por outro lado, a maioria relatou consumir excesso de alimentos do grupo dos feijões, carnes, óleos e gorduras e açúcares e doces (Tabela 3).

A avaliação da ingestão de nutrientes específicos mostrou que a maioria das pacientes tinha baixo consumo de cálcio, vitaminas B6, B12 e A. (Tabela 4). O consumo de álcool foi relatado por $25 \%(8 / 32)$ das pacientes, sendo que destas $62,5 \%$ $(5 / 8)$ consumiam mais que $10 \mathrm{~g}$ por dia.

Foram avaliadas possíveis associações entre diferentes componentes da dieta, IASad e a agressividade do câncer de mama, entretanto nenhuma associação foi encontrada.

Tabela 4. Frequência da ingestão de nutrientes específicos avaliado pelo QFA de mulheres com câncer de mama, atendidas pelo Serviço de Mastologia do Hospital das Clínicas, da UFMG, nos anos 2010-2011 ( $\mathrm{n}=31)$.

\begin{tabular}{|c|c|}
\hline Nutrientes & n (\%) \\
\hline \multicolumn{2}{|l|}{ Carboidrato (\%) } \\
\hline$<45$ & $1(3,2 \%)$ \\
\hline $45-65$ & $22(70,9 \%)$ \\
\hline$\geq 65$ & $8(25,8 \%)$ \\
\hline \multicolumn{2}{|l|}{ Proteína (\%) } \\
\hline$<10$ & $2(6,4 \%)$ \\
\hline $10-35$ & $29(93,5 \%)$ \\
\hline \multicolumn{2}{|l|}{ Lipídeos (\%) } \\
\hline$<20$ & $7(22,5 \%)$ \\
\hline $20-35$ & $23(74,1 \%)$ \\
\hline$\geq 35$ & $1(3,2 \%)$ \\
\hline \multicolumn{2}{|l|}{ Fibra $(\mathrm{g})$} \\
\hline$<21$ & $0(0 \%)$ \\
\hline$\geq 21$ & $31(100 \%)$ \\
\hline \multicolumn{2}{|l|}{ Ferro (mg) } \\
\hline$<5$ & $0(0 \%)$ \\
\hline$\geq 5$ & $31(31 \%)$ \\
\hline \multicolumn{2}{|l|}{ Cálcio (mg) } \\
\hline$<1000$ & $24(77,4 \%)$ \\
\hline$\geq 1000$ & $7(22,5 \%)$ \\
\hline \multicolumn{2}{|l|}{ Vitamina C (mg) } \\
\hline$<60$ & $10(32,2 \%)$ \\
\hline$\geq 60$ & $21(67,7 \%)$ \\
\hline \multicolumn{2}{|l|}{ Vitamina B6 (mg) } \\
\hline$<1,3$ & $19(61,2 \%)$ \\
\hline$\geq 1,3$ & $12(38,7 \%)$ \\
\hline \multicolumn{2}{|l|}{ Vitamina B12 (mg) } \\
\hline$<2$ & $28(90,3 \%)$ \\
\hline$\geq 2$ & $3(9,6 \%)$ \\
\hline \multicolumn{2}{|l|}{ Vitamina $\mathrm{A}(\mu \mathrm{g})$} \\
\hline$<500$ & $28(90,3 \%)$ \\
\hline$\geq 500$ & $3(90,3 \%)$ \\
\hline
\end{tabular}

\section{Discussão}

No presente trabalho a população estudada foi em sua maioria de mulheres negras e pardas (68,75\%). Ao avaliar o nível educacional, 43,74\%, possuíam sete anos ou menos de escolaridade. Houve maior prevalência de carcinoma ductal invasivo, de grau histológico tipo II em estadio II ou III. Os resultados obtidos do perfil imunohistoquímico foram diversificados. Mulheres obesas tendem a ser diagnosticadas em estadios mais avançados da doença, com tumores maiores, envolvimento dos linfonodos e grau histológico III ${ }^{22}$.

$O$ câncer de mama positivo para HER2 possui incidência habitual de $18-20 \%{ }^{23}$. A positividade para HER-2 está associada com maior agressividade da doença, constituindo um indicador de comportamento clínico mais agressivo e com pior resposta às terapêuticas convencionais ${ }^{24}$.

Aproximadamente $80 \%$ dos cânceres de mama são positivos para receptor de estrógeno ${ }^{25}$, o que está de acordo com o presente trabalho, no qual $60 \%$ dos casos mostraram positividade para o marcador. A diminuição da expressão de receptores hormonais tem valor prognóstico nos tempos livres de doença e de metástases e na sobrevida global ${ }^{26}$.

Existem evidências de que a adiposidade corporal aumentada é fator de risco para o desenvolvimento do câncer de mama. A relação entre excesso de peso e diagnóstico de câncer de mama em estádios avançados tem sido demonstrada independentemente do estado menopausal ${ }^{27}$. Assim, excesso de gordura corporal, obesidade e circunferência da cintura elevada têm sido associados ao aumento do risco de desenvolver a doen$\mathrm{ça}^{2}$. A maioria das mulheres avaliadas no presente trabalho apresentou excesso de peso, valores aumentados de circunferência de cintura e excesso de gordura corporal, como verificado por outros autores ${ }^{28,29}$. Contudo, no presente estudo não houve associações entre IMC, CC, \%GC e o estadiamento e grau histólogico do tumor. Esses resultados assemelham-se ao do estudo de Amaral et al., no qual 48\% das mulheres com câncer de mama apresentaram excesso de peso, $62 \%$ das pacientes apresentaram CC $>88 \mathrm{~cm}$ e $89 \%$ tinham excesso de gordura corporal. Neste mesmo estudo foram detectadas associações pela análise de variância multivariada entre IMC, CC, $\% \mathrm{GC}$, histologia e maior agressividade do tumor $(\mathrm{p}<0,05)$, sendo que todas as variáveis pioraram com o aumento da idade ${ }^{2}$. Na população brasileira o excesso de peso corporal é mais prevalente em mulheres com câncer de mama do que na 
população em geral ${ }^{30,31}$. O estudo de Felden e Figueiredo $^{32}$ associou a distribuição da gordura corporal e o câncer de mama em mulheres do Rio Grande do Sul e verificou que o acúmulo de gordura na parte superior do corpo $(\mathrm{CC}>88 \mathrm{~cm})$ é um preditor dessa neoplasia, especialmente em mulheres na pré-menopausa.

As pesquisas de consumo alimentar constituem instrumentos eficazes e de baixo custo para obtenção de informações sobre as características da alimentação de grande parte da população, mas há as limitações de cada método ${ }^{33}$. Alimentação saudável composta por maior consumo de vegetais e frutas (cinco porções por dia) e menor ingestão de gordura está associada com redução nos níveis séricos de estrógeno ${ }^{34}$. Dados da Pesquisa de Orçamentos Familiares (POF 2008-2009) demonstraram que $82 \%$ dos brasileiros consomem excesso de gordura saturada, $61 \%$ excesso de açúcar e $68 \%$ baixa quantidade de fibras ${ }^{35}$. No presente trabalho, mais de $80 \%$ dos participantes tiveram baixo consumo de frutas, legumes e verduras em relação aos valores recomendados. O consumo deste grupo de alimentos, isolados ou associados, é um fator de proteção para o desenvolvimento de síndrome metabólica, diabetes mellitus, doenças cardiovasculares, dislipidemias e outras causas de mortes, porém a maioria das populações não tem alcançado a ingestão recomendada, sendo o incentivo a melhor forma de estimular o consumo, devendo ser feito para todos os indivíduos ${ }^{36}$. Do mesmo modo, Amaral et al. encontraram baixo consumo de vegetais, cereais integrais, fibras, fitoquímicos, alimentos ricos em ácidos graxos poliinsaturados (ômega -3) e alto consumo de gordura saturada por mulheres com câncer de mama $^{2}$. Elevada ingestão de gordura pode estar associada positivamente com aumento no risco de desenvolver câncer de mama em mulheres ${ }^{37} \mathrm{e}$ maior prevalência de tumores positivos para receptores hormonais na pré e pós-menopausa ${ }^{37,38}$.

A qualidade da alimentação referente aos grupos alimentares (avaliada pelo IASad) ou a nutrientes específicos (avaliado pelo QFA), refletiu baixo consumo de alimentos fontes de cálcio e de vitaminas A, B6 e B12, enquanto que o de vitamina $\mathrm{C}$ foi adequado para a maioria das pacientes avaliadas. Trabalhos mostram a associação positiva entre baixo consumo de cálcio ${ }^{39}$ e vitamina $\mathrm{A}^{40}$ e maior risco para câncer de mama. Chen et al. mostraram que o consumo elevado de vitamina $\mathrm{D}$ e cálcio possui efeito quimiopreventivo para câncer de mama ${ }^{40}$. As vitaminas A e C parecem agir sinergisticamente na inibição da proliferação de células de câncer de mama, por induzir a expressão de moléculas envolvidas na diferenciação, inibição da proliferação, apoptose, antioxidação, regulação do ciclo celular e reparo do $\mathrm{DNA}^{41}$. Fulan et al. ${ }^{42}$ mostraram que o consumo de vitamina A parece reduzir o risco de câncer de mama. $\mathrm{O}$ consumo elevado de vitamina C (suplementação acima de $1000 \mathrm{mg} / \mathrm{dia}$ ou mais que cinco porções de frutas, legumes e verduras/dia) parece ter um papel protetor, mas os resultados ainda são inconclusivos ${ }^{43}$. Mulheres com câncer de mama apresentaram baixos níveis séricos de beta-caroteno, quando comparadas com mulheres saudáveis ${ }^{44}$.

Em conclusão, visto que o câncer de mama possui alta prevalência na população mundial, acredita-se que medidas de promoção da saúde devem ser incentivadas, com foco na prevenção dos fatores de risco para o desenvolvimento da doença. Verifica-se que maior prevalência de sobrepeso ou obesidade juntamente com a inadequação da alimentação das pacientes avaliadas demonstra a necessidade da realização de acompanhamento e educação nutricional, a fim de melhorar o prognóstico e evitar o desenvolvimento da doença.

\section{Colaboradores}

DR Oliveira, LC Campos, JA Leal, EV Sampaio, GD Cassali e ESC Carvalho participaram igualmente de todas as etapas de elaboração do artigo. 


\section{Referências}

1. Emaus A, Marit BV, Steinar T, Finstad SE, Selmer R, Furberg A, Bernstein L, Schlichting E, Thune I. Metabolic profile, physical activity, and mortality in breast cancer patients. Breast Cancer Res Treat 2010; 121(3):651-660.

2. Amaral P, Miguel R, Mehdad A, Cruz C, Monteiro Grillo I, Camilo A, Ravasco P. Body fat and poor diet in breast cancer women. Nutr Hosp 2010; 25(3): 456-461.

3. Vance V, Mourtzakis M, Mccargar L, Hanning R. Weight gain in breast cancer survivors: prevalence, pattern and health consequences. Obes Rev 2011; 12(4):282-294.

4. Patterson RE, Cadmus LA, Emond JA, Pierce JP. Physical activity, diet, adiposity and female breast cancer prognosis: A review of the epidemiologic literature. Maturitas 2010; 66(1):5-15.

5. Padilha PC, Pinheiro RL. Alimentos Funcionais no Câncer de Mama. Rev Bras Cancerol 2004; 50(3):251260.

6. Buckland G, Travier N, Cottet V, González CA, LujánBarroso L, Agudo A, Trichopoulou A, Lagiou P, Trichopoulos D, Peeters PH, May A, Bueno-de-Mesquita HB, Bvan Duijnhoven FJ, Key TJ, Allen N, Khaw KT, Wareham N, Romieu I, McCormack V, Boutron-Ruault M, Clavel-Chapelon F, Panico S, Agnoli C, Palli D, Tumino R, Vineis P, Amiano P, Barricarte A, Rodríguez L, Sanchez MJ, Chirlaque MD, Kaaks R, Teucher B, Boeing H, Bergmann MM, Overvad K, Dahm CC, Tjønneland A, Olsen A, Manjer J, Wirfält E, Hallmans G, Johansson I, Lund E, Hjartåker A, Skeie G, Vergnaud AC, Norat T, Romaguera D, Riboli E. Adherence to the mediterranean diet and risk of breast cancer in the European prospective investigation into cancer and nutrition cohort study. Int J Cancer 2013; 132(12):2918-2927.

7. Lindgren J, Dorgan J, Savage-Williams J, Coffman D, Hartman T. Diet across the Lifespan and the Association with Breast Density in Adulthood. Int J Breast Cancer 2013; 2013:808317.

8. Piêta B, Chmaj-Wierzchowska K, Opala T. Life style and risk of development of breast and ovarian cancer. Ann Agric Environ Med 2012; 19(3):379-384.

9. Instituto Nacional de Câncer (INCA). TNM: classificação de tumores malignos. Rio de Janeiro: INCA; 2004.

10. Instituto Nacional de Câncer (INCA). Estimativa 2010: incidência de câncer no Brasil. Rio de Janeiro: INCA; 2009.

11. Zaboto CB, Vianna RPT, Gil MF. Registro Fotográfico para Inquéritos Dietéticos: Utensílios e Porções. Campinas: Unicamp; 1996.

12. Salvo VL, Gimeno SG. Reproducibility and validity of a food frequency questionnaire. Rev Saude Publica 2002; 36(4):505-512.

13. Mota JF, Rinaldi AEN, Pereira AF, Maestá N, Scarpin MM, Burini RC. Adaptação do índice de alimentação saudável ao guia alimentar da população brasileira. Rev Nutr 2008; 21(5):545-552.

14. Universidade Estadual de Campinas (Unicamp). Núcleo de Estudos e Pesquisas em Alimentação. Tabela brasileira de composição de alimentos - TACO. $2^{\text {a }}$ ed. Campinas: Nepa, Unicamp; 2006.
15. Phillipi ST. Tabela de Composição de Alimentos: suporte para decisão nutricional. São Paulo: Coronário; 2002.

16. Pacheco M. Tabela de equivalentes, medidas caseiras e composição química dos alimentos. Rio de Janeiro: Rubio; 2006.

17. National Research Council. Dietary Reference Intakes: Applications in Dietary Assessment. Washington: National Academy Press; 2000.

18. World Health Organization (WHO). Obesity: preventing and managing the global epidemic - report of a WHO consultation on obesity. Geneva: WHO; 2000.

9. World Health Organization (WHO). Obesity: preventing and managing the global epidemic. Geneva: WHO; 1998.

20. Durnin JVGA, Wormersley J. Body fat assessed from total body density and its estimation from skinfold thickness: measurements on 481 men and women aged from 16 to 72 years. Br J Nutr 1974; 32(1):77-97.

21. Lohman TG. Advances in body composition assessment: current issues in exercises science. Illinois: Human Kinetic Publisher; 1992.

22. Ewertz M, Jensen M, Gunnarsdo’Ttir K, Højris I, Jakobsen EH, Nielsen D, Stenbygaard LE, Tange UB, Cold S. Effect of Obesity on Prognosis After EarlyStage Breast Cancer. J Clin Oncol 2011; 29(1):25-31.

23. Wolff AC, Hammond, MEH, Schwartz JN, Hagerty KL, Allred DG, Cote RJ, Dowsett M, Fitzgibbons PL, Hanna WM, Langer A, McShane LM, Paik S, Pegram MD, Perez EA, Press MF, Rhodes A, Sturgeon C, Taube SE, Tubbs R, Vance GH, van de Vijver M, Wheeler TM, Hayes DF; American Society of Clinical Oncology; College of American Pathologists. Pathologists Guideline Recommendations for Human Epidermal Growth Factor Receptor 2 Testing in Breast Cancer. J Clin Oncol 2007; 25(1):118-145.

24. Yamashita H, Iwase H, Toyama T, Takahash S, Sugiura H, Yoshimoto N, Endo Y, Fujii Y, Kobayashi S. Estrogen receptor-positive breast cancer in Japanese women: trends in incidence, characteristics, and prognosis. Ann Oncol 2011; 22(6):1318-1325.

25. Perks CM, Holly JMP. Hormonal Mechanisms Underlying the Relationship Between Obesity and Breast Cancer. Endocrinol Metab Clin North Am 2011; 40(3):485-507. 
26. Yang XR, Chang-Claude J, Goode EL, Couch FJ, Nevanlinna H, Milne RL, Gaudet M, Schmidt MK, Broeks A, Cox A, Fasching PA, Hein R, Spurdle AB, Blows F, Driver K, Flesch-Janys D, Heinz J, Sinn P, Vrieling A, Heikkinen T, Aittomäki K, Heikkilä P, Blomqvist C, Lissowska J, Peplonska B, Chanock S, Figueroa J, Brinton L, Hall P, Czene K, Humphreys $K$, Darabi H, Liu J, Van 't Veer LJ, van Leeuwen FE, Andrulis IL, Glendon G, Knight JA, Mulligan AM, O'Malley FP, Weerasooriya N, John EM, Beckmann MW, Hartmann A, Weihbrecht SB, Wachter DL, Jud SM, Loehberg CR, Baglietto L, English DR, Giles GG, McLean CA, Severi G, Lambrechts D, Vandorpe T, Weltens C, Paridaens R, Smeets A, Neven P, Wildiers H, Wang X, Olson JE, Cafourek V, Fredericksen Z, Kosel M, Vachon C, Cramp HE, Connley D, Cross SS, Balasubramanian SP, Reed MW, Dörk T, Bremer M, Meyer A, Karstens JH, Ay A, Park-Simon TW, Hillemanns P, Arias Pérez JI, Menéndez Rodríguez P, Zamora P, Benítez J, Ko YD, Fischer HP, Hamann U, Pesch B, Brüning T, Justenhoven C, Brauch H, Eccles DM, Tapper WJ, Gerty SM, Sawyer EJ, Tomlinson IP, Jones A, Kerin M, Miller N, McInerney N, Anton-Culver H, Ziogas A, Shen CY, Hsiung CN, Wu PE, Yang SL, Yu JC, Chen ST, Hsu GC, Haiman CA, Henderson BE, Le Marchand L, Kolonel LN, Lindblom A, Margolin S, Jakubowska A, Lubiñski J, Huzarski T, Byrski T, Górski B, Gronwald J, Hooning MJ, Hollestelle A, van den Ouweland AM, Jager A, Kriege M, Tilanus-Linthorst MM, Collée M, Wang-Gohrke S, Pylkäs K, Jukkola-Vuorinen A, Mononen K, Grip M, Hirvikoski P, Winqvist R, Mannermaa A, Kosma VM, Kauppinen J, Kataja V, Auvinen P, Soini Y, Sironen R, Bojesen SE, Ørsted DD, Kaur-Knudsen D, Flyger H, Nordestgaard BG, Holland H, Chenevix-Trench G, Manoukian S, Barile M, Radice P, Hankinson SE, Hunter DJ, Tamimi R, Sangrajrang S, Brennan P, McKay J, Odefrey F, Gaborieau V, Devilee P, Huijts PE, Tollenaar RA, Seynaeve C, Dite GS, Apicella C, Hopper JL, Hammet F, Tsimiklis H, Smith LD, Southey MC, Humphreys MK, Easton D, Pharoah P, Sherman ME, Garcia-Closas M. Associations of Breast Cancer Risk Factors With Tumor Subtypes: A Pooled Analysis From the Breast Cancer Association Consortium Studies. J Natl Cancer Inst 2011; 103(3):250-263.

27. Carmichael AR. Obesity and prognosis of breast cancer. Obes Rev 2006; 7(4):333-340.

28. Khan N, Afaq F, Mukhtar H. Lifestyle as risk factor for cancer: Evidence from human studies. Cancer Lett 2010; 293(2):133-143.

29. World Cancer Research Fund (WCRF). Food, Nutrition, Physical Activity and the Prevention of Cancer: a Global Perspective. Washington: American Institute for Cancer Research; 2007.

30. Matos JC, Pelloso SM, Carvalho MDB. Prevalência de fatores de risco para o câncer de mama no município de Maringá, Paraná. Rev. Latino-Am. Enfermagem 2010; 18(3):57-64.

31. Rubin BA, Stein AT, Zelmanowicz AM, Rosa DD. Perfil Antropométrico e Conhecimento Nutricional de Mulheres Sobreviventes de Câncer de Mama do Sul do Brasil. Rev Bras Cancerol 2010; 56(3):303309.
32. Felden JBB, Figueiredo ACL. Distribuição da gordura corporal e câncer de mama: um estudo de caso-controle no Sul do Brasil. Cien Saude Colet 2011; 16(5):2425-2433.

33. Fisberg RM, Colucci ACA, Morimoto JM, Marchioni DML. Food frequency questionnaire for adults from a population-based study. Rev Saude Publica 2008; 42(3):550-554.

34. Brennan SF, Cantwell MM, Cardwell CR, Velentzis LS, Woodside JV. Dietary patterns and breast cancer risk: a systematic review and meta-analysis. $A m$ J Clin Nutr 2010; 91(5):1294-1302.

35. Instituto Brasileiro de Geografia e Estatística (IBGE). Pesquisa de Orçamentos Familiares 2008-2009. Rio de Janeiro: IBGE; 2010. [Internet]. [acessado 2011 nov 3]. Disponível em: http://www.ibge.gov.br

36. Castanho GKF, Marsola FCM, Kátia CP, Nicola M, Moreto F, Burini RC. Consumo de frutas, verduras e legumes associado à Síndrome Metabólica e seus componentes em amostra populacional adulta. Cien Saude Colet 2013; 18(2):385-392.

37. Turner LB. A Meta-analysis of Fat Intake, Reproduction, and Breast Cancer Risk: An Evolutionary Perspective. Am J Hum Biol 2011; 23(5):601-608.

38. Cho E, Spieglman D, Hunter DJ, Chen WY, Stampfer MJ, Colditz GA, Willett WC. Premenopausal fat intake and risk of breast cancer. J Natl Cancer Inst 2003; 95(14):1079-1085.

39. Kushi LH, Potter JD, Bostick RM, Drinkard CR, Sellers TA, Gapstur SM, Cerhan JR, Folsom AR. Dietary fat and risk of breast cancer according to hormone receptor status. Cancer Epidemiol Biomarkers Prev 1995; 4(1):11-19.

40. Chen P, Hu P, Xie D, Qin Y, Wang F, Wang H. Metaanalysis of vitamin $\mathrm{D}$, calcium and the prevention of breast cancer. Breast Cancer Res Treat 2010; 121(2):469-477.

41. Kim K, Pie J, Park J, Park Y, Kim H, Kim M. Retinoic acid and ascorbic acid act synergistically in inhibiting human breast cancer cell proliferation. $J$ Nutr Biochem 2006; 17(7):454-462.

42. Fulan H, Changxing J, Baina WY, Wencui Z, Chunqing L, Fan W, Dandan L, Dianjun S, Tong W, Da P, Yashuang Z. Retinol, vitamins A, C, and E and breast cancer risk: a meta-analysis and meta-regression. Cancer Causes Control 2011; 22(10):1383-1396.

43. Hutchinson J, Burley VJ, Greenwood DC, Thomas JD, Cade JE. High-dose vitamin C supplement use is associated with self-reported histories of breast cancer and other illnesses in the UK Women's Cohort Study. Public Health Nutr 2011; 14(5):768-777.

44. Nkondjock A, Ghadirian P. Intake of specific carotenoids and essential fatty acids and breast cancer risk in Montreal, Canada. Am J Clin Nutr 2004; 79(5):857-864.

Artigo apresentado em 15/03/2013

Aprovado em 25/03/2013

Versão final apresentada em 26/03/2013 